\title{
«¿16 céntimos a cambio de aguas ácidas?». Respuestas políticas a la expansión minera en Cajamarca
}

\author{
Mauricio Zavaleta*
}

* Mauricio Zavaleta es licenciado en Ciencia Política y Gobierno por la Pontificia Universidad Católica del Perú. Correo electrónico: mauriciozavaleta@gmail.com

Fecha de recepción: 19/03/18. Fecha de aceptación: 13/12/18 


\title{
«¿16 céntimos a cambio de aguas ácidas?». Respuestas políticas a la expansión minera en Cajamarca
}

\section{RESUMEN}

Durante dos décadas, Cajamarca albergó una de las minas de oro más productivas del mundo. Entre 1992, cuando se iniciaron las operaciones del yacimiento Yanacocha, y 2011, cuando fue suspendido el proyecto expansivo más importante de la empresa operadora, la ampliación de las actividades extractivas ha sido fuente de controversia. Solo entre 2004 y 2011, tres conflictos alcanzaron relevancia nacional en Cajamarca: Quilish (2004), Combayo (2006) y Minas Conga (2011). Este continuo permite analizar las diferentes «respuestas» de la población —especialmente aquella de las zonas rurales - a la minería. El artículo propone que las respuestas campesinas han estado orientadas a la negociación de beneficios que compensen la afectación de los recursos naturales y permitan a las localidades acceder, aunque de manera indirecta y limitada, a las ganancias producidas por la industria. Sin embargo, cuando los acuerdos logrados con la empresa no son cumplidos o la población percibe que la afectación a los medios de subsistencia es mayor que los beneficios recibidos, se generan incentivos para la movilización. En ese contexto, emergieron diferentes coaliciones entre caseríos rurales y organizaciones urbanas que, aunque efímeras en términos organizativos, promovieron movilizaciones en contra de los proyectos de expansión de Yanacocha con notable efectividad.

Palabras clave: conflictividad social, minería, protesta, coaliciones, Cajamarca, Conga.

\section{«16 cents in exchange for acid waters?». Responses to the mining expansion in Cajamarca}

\begin{abstract}
For decades, Cajamarca has housed one of the most productive gold mines in the world. Between 1992, when the operations of the Yanacocha deposits began, until 2011, when the most important expansion project of the operating company was suspended, extractive activities have been a source of controversy. Only between 2004 and 2011 three conflicts reached national relevance in Cajamarca: Quilish (2004), Combayo (2006) and Minas Conga (2011). This continuum allows analyzing different types of «responses» — especially from rural areas - to mining. The article proposes that the peasant responses have been oriented to the negotiation of benefits that compensate the affectation of natural resources and allow the localities to accede, albeit in an indirect and limited way, to the profits produced by the industry. However, when the agreements reached with the company are not met or the population perceives that the impact on the means of subsistence is greater than the benefits received, incentives are generated for political mobilization. In this context, different coalitions had emerged between rural villages and urban organizations that, though ephemeral in organizational terms, promoted mobilizations against Yanacocha's expansion projects with remarkable effectiveness.
\end{abstract}

Keywords: social conflict, mining, protest, coalitions, Cajamarca, Conga. 


\section{INTRODUCCIÓN $^{1}$}

El Perú fue uno de los países que más se benefició, desde un punto de vista macroeconómico, con el alza del precio de los commodities de la primera década del siglo XX (Monaldi, 2014). Entre 2001 y 2011, la tasa de crecimiento anual del PBI nacional fue de 6,3\%, lo cual auspició un dramático descenso en los niveles de pobreza. Asimismo, la periferia del país experimentó cambios significativos: un amplio número de regiones creció a tasas porcentuales que sobrepasaban o igualaban a la capital metropolitana — un hecho sin precedentes en la historia contemporánea-, mientras que el presupuesto agregado de los gobiernos subnacionales pasó de 81 millones de soles en 2001 a 4500 millones en 2012 (De la Flor, 2014).

En este contexto, surgió una serie de conflictos vinculados con la extracción de recursos naturales. De acuerdo con la Defensoría del Pueblo, el 52\% de los conflictos registrados entre 2009 y 2015 en el país eran producto centralmente de actividades mineras o hidrocaburíferas (Defensoría del Pueblo, 2017). En los dos trabajos más exhaustivos sobre la materia, Javier Arellano-Yanguas (2014) y Moisés Arce (2015) sostienen que los conflictos en torno a la industria minera pueden clasificarse en dos categorías: (i) aquellos que se encuentran en contra de las actividades extractivas per se (conflictos socioambientales), y (ii) aquellos vinculados con el incumplimiento de acuerdos y demanda de servicios (conflictos redistributivos). Los primeros estarían impulsados por la defensa de los medios de subsistencia de la población rural, mientras que los segundos buscarían acceder a los beneficios económicos derivados de la extracción ${ }^{2}$.

No obstante, en muchos de los conflictos esta naturaleza no se encuentra claramente delimitada y se combinan demandas relacionadas con los recursos naturales (socioambientales) y con los beneficios materiales de la inversión (redistributivas). Los conflictos expansivos - es decir, aquellos que comprometen la ampliación de las fronteras de la zona de extracción — ilustran de buena manera esta ambigüedad. En el presente artículo sostenemos que este tipo de conflictos permite desarrollar teorías sobre la racionalidad de la población directamente impactada por las actividades mineras y la configuración de coaliciones políticas que llevan el conflicto a escala regional y, eventualmente, nacional e internacional.

Cajamarca, en la sierra norte del Perú, admite observar el desarrollo de los conflictos expansivos de manera longitudinal. En 1992 fue inaugurado en la región

\footnotetext{
1 Este artículo ha sido hecho sobre la base de un documento de trabajo para el Departamento de Ciencias Sociales de la Pontificia Universidad Católica del Perú (2014).

2 La nomenclatura de ambos autores difiere: Arellano-Yanguas (2014) distingue entre «conflictos antimineros» y "conflictos redistributivos», mientras que Arce (2015) hace referencia a «demanda de derechos» $\mathrm{y}$ «demanda de servicios».
} 
el proyecto minero más importante del país en décadas: Yanacocha, operado por la empresa homónima ${ }^{3}$. Si bien durante la primera década de actividades de la compañía su vinculación con las áreas rurales de Cajamarca estuvo marcada por conflictos de baja intensidad que no ponían en riesgo sus operaciones, a partir de 2004, con las movilizaciones en contra de la expansión de la mina a un cerro ubicado a pocos kilómetros de la capital departamental (Quilish), los proyectos de Yanacocha fueron motivo de alta conflictividad.

En tal sentido, analizar el caso de Cajamarca permite mirar con mayor profundidad los antecedentes de cada episodio de movilización. Desde su ingreso a la región, Yanacocha estableció como política de relacionamiento comunitario la implementación de programas sociales privados y la contratación de empresas subsidiarias comunales formadas por quienes habían vendido sus tierras a la minera. Sin embargo, cuando Yanacocha no fue capaz de contratar los servicios de estas empresas de manera constante o incumplió compromisos con la población, se inició un ciclo de desavenencias potenciadas por accidentes ambientales ocasionados por la compañía ${ }^{4}$. Paulatinamente se creó la percepción de que los beneficios recibidos por la población eran menores que la afectación de los medios tradicionales de subsistencia rural, lo cual incrementó considerablemente los costos de transacción en las negociaciones entre la empresa y las comunidades. Luego de Quilish, al menos cinco proyectos expansivos fueron bloqueados por movilizaciones sociales: San Cirilo (2005), Carachugo II (2006), La Quinua Sur (2007), Solitario (2009) y Minas Conga (2011).

Estas movilizaciones fueron sostenidas por coaliciones integradas por actores rurales y urbanos que se reconfiguraron en cada episodio de protesta: nuevos líderes y aparatos surgieron para cada nuevo período de acción política. Antes que sus soportes organizativos, la fortaleza de estas coaliciones promotoras fue la difusión de un discurso ambientalista forjado con el tiempo, el cual hacía responsable a Yanacocha de afectar el completo ecosistema de la región. Este discurso, que rápidamente derivaría en una controversia entre expertos de diferentes filiaciones (Zavaleta, 2013; Li, 2017; Yrrivaren, 2018) no tuvo un correlato organizativo, salvo

\footnotetext{
3 Yanacocha SRL, constituida por capitales estadounidenses (Newmont Mining Company, 51,35\%), peruanos (Compañía de Minas Buenaventura, 43,65\%) y la participación de la International Finance Corporation (IFC) del Banco Mundial (5\%). En 2018, Sumitomo Corporation adquirió el porcentaje de las acciones que correspondían a la IFC.

4 Los accidentes involucraron el derrame de sustancias tóxicas, descuido en el tratamiento de los relaves y muerte de peces. De especial relevancia fue el derrame de mercurio en los centros poblados de San Juan, Magdalena y Choropampa, por el cual fueron contaminadas alrededor de 1200 personas. De acuerdo con Irwin y Gallagher (2012), Yanacocha es la empresa que ha pagado mayores montos en multas ambientales por años de operación.
} 
el pasajero Comando Unitario de Lucha (CUL), agrupación que acogió a los diferentes frentes que mostraron su oposición a la expansión minera.

El presente artículo recoge información de los casos de Combayo (2006) y, especialmente, Conga (2011-2012), dos proyectos de carácter expansivo que fueron suspendidos como resultado de episodios de protesta. El análisis está basado en documentos, veintitrés entrevistas a informantes clave y observación participante de las movilizaciones en contra del proyecto Conga en la ciudad de Cajamarca en junio de 2012. Sin embargo, es importante mencionar que una mayor evidencia empírica en favor del desarrollo teórico relativo a la toma de decisiones de la población rural requiere la construcción de experimentos sociales que brinde resultados más robustos. En tal sentido, el presente trabajo es una primera aproximación teórica desde técnicas cualitativas tradicionales de recojo de información.

El artículo se divide en tres partes. La primera presenta brevemente el debate reciente en torno la conflictividad social vinculada con las industrias extractivas en el Perú y discute la relevancia teórica del caso de Cajamarca en su conjunto. La segunda sección identifica las respuestas rurales a la industria minera en la región, con especial énfasis en las respuestas políticas. Tomando documentos producidos en el marco de los conflictos de Combayo y Conga, busca aproximarse a los parámetros de negociación de la población rural con Yanacocha. El tercer apartado discute la formación de coaliciones políticas entre actores urbanos y rurales en contra de las actividades de la empresa. Entre 2011 y 2012, una nueva coalición de este tipo sostuvo las protestas en contra del proyecto Conga por un lapso de diez meses. Finalmente, se presentan las conclusiones.

\section{Minería y CONFLiCTIVIDAD SOCIAL}

La conflictividad social en torno a las industrias extractivas ha generado un gran interés académico. ¿Cuáles son las causas de la conflictividad en torno, principalmente, a las empresas mineras? A través de un análisis de casos agregados, Arellano-Yaguas (2014) propone que, antes que la afectación a los medios de subsistencia resaltados en la mayoría de estudios de caso ${ }^{5}$, las movilizaciones en torno a las actividades extractivas se explican por estrategias de apropiación de la renta minera por diferentes actores. Así, propone que este tipo de estrategias de negociación habrían sido incentivadas por el notable incremento de los presupuestos destinados a programas de responsabilidad social corporativa y al establecimiento de transferencias fiscales a los gobiernos subnacionales, en particular en la figura

\footnotetext{
De acuerdo con Perla (2017), cerca del 70\% de la literatura producida entre 2000 y 2010 estaba relacionada con cinco casos de estudio.
} 
del canon minero. Para el autor, la implementación de este conjunto de estrategias redujo el número de conflictos sociales genuinamente «antimineros»; sin embargo, aumentó considerablemente el número total de conflictos «redistributivos».

Más atento a las variables políticas, Moisés Arce (2015), a través del análisis de una base de datos distinta, propone que los factores políticos tienen mayor capacidad explicativa sobre el origen de los conflictos en torno a las industrias extractivas que el flujo de recursos. Si bien el monto del canon minero es una variable estadísticamente significativa en su análisis, la controversia en torno a su distribución debe ser entendida por la fragmentación de la política regional. La alta fragmentación política en un contexto de apertura democrática y descentralización impide establecer "mecanismos de respuesta" a las demandas de los ciudadanos y "crea incentivos para el uso de estrategias de confrontación y perturbación con las cuales incidir en quienes gobiernan» (Arce, 2015, p. 192).

No obstante, tanto los análisis de casos como aquellos basados en evidencia estadística tienden a perder de vista los diferentes grados de conflicto: desde su completa ausencia hasta la protesta pública en capitales provinciales ${ }^{6}$. En este enorme continuo existe una heterogeneidad de encuentros entre empresas mineras y poblaciones locales que, como afirma Cecilia Perla, «no es de abierta confrontación, ni mucho menos de total rechazo, sino, más bien, un largo proceso salpicado con algunas escaramuzas, y marcado por continuas negociaciones, desconfianza y falta de capacidades, y por tantear los límites entre lo que se puede demandar y se puede ofrecer» (2017, p. 122). En tal sentido, en la medida que los trabajos académicos se han focalizado en los episodios de protesta, es limitada la información disponible sobre la gestación de la conflictividad en los espacios donde las empresas y los ciudadanos interactúan de forma cotidiana y sin intermediarios.

En ese sentido, la historia reciente de Cajamarca nos brinda la posibilidad de observar el «largo proceso» de cambio en los patrones de relacionamiento de los ciudadanos rurales con una gran empresa minera, lo que, siguiendo un clásico trabajo de Robert Bates (1976) llamaré respuestas rurales a la industrialización. Sostengo que caracterizar los conflictos mineros como «socioambientales» o «redistributivos» como proponen Arellano-Yanguas y Arce- pierde de vista los conflictos generados por la expansión de las actividades mineras, es decir, aquellos conflictos resultantes de la ampliación física de un determinado yacimiento o la implementación de nuevos proyectos en zonas adyacentes por una misma empresa.

Entre 2004 y 2011 tres conflictos alcanzaron relevancia nacional en Cajamarca, los cuales involucraban diferentes proyectos de Minera Yanacocha: Quilish (2004),

6 De acuerdo con Perla (2017, p. 11), en 2010 solo 77 (20\%) de las 443 unidades mineras en exploración y explotación reportaban algún tipo de conflicto. 
Combayo (2006) y Minas Conga (2011), mientras que los «microconflictos» colaterales a la actividad minera también han sido recurrentes: solo entre 2008 y 2011 , De Echave y Diez (2013, p. 87) registran doce conflictos de mediana relevancia que derivaron en hechos violentos. Debido a que este tipo de conflictos muestran mayor complejidad y son difíciles de encasillar en la dicotomía planteada por Arce (2015) y Arellano-Yanguas (2014), son de especial relevancia para analizar la racionalidad y estrategia de los actores en disputa.

Si bien mayores montos en el gasto social de las empresas y el flujo de canon minero parecen ser poderosos alicientes para la movilización, queda por explorar de qué manera los ciudadanos establecen estrategias de negociación a lo largo del tiempo o cómo valoran el acceso a los medios de subsistencia. Sostengo que echar luz sobre esta ambivalencia permite hacer contribuciones teóricas relevantes acerca de la racionalidad de la población rural y sus «respuestas» a las industrias extractivas, así como la formación de "coaliciones promotoras» que facilitan la escalada del conflicto en los ámbitos regional o nacional ${ }^{7}$. En el caso de Cajamarca, esta intermediación ha sido efectuada por actores urbanos que lograron establecer vínculos con los alcaldes de centros poblados o tenientes gobernadores, pero carentes de una organización estructurada con vida orgánica más allá del período de movilización.

\section{Respuestas RURAles a la industria minera en CajamarCa (Historia mínima)}

Yanacocha utiliza en sus yacimientos un método de extracción por lixiviación de cianuro a través del cual toneladas de tierra removida son rociadas con una solución que absorbe el oro para luego separarlo del fluido (Bury, 2007a). Como otros procesos similares, la extracción de oro utilizada por Yanacocha requiere, además de una inversión intensiva en capital, mano de obra calificada y el uso de grandes extensiones de terreno y acceso a fuentes de agua. Debido a ello, la población local solo accede de manera parcial y temporal al empleo ofrecido por las empresas, generalmente durante el período de construcción (Bebbington, 2013). La sustitución de mano de obra intensiva por nuevas tecnologías de extracción ha sido uno de los cambios de mayor relevancia para comprender los conflictos en torno a las industrias extractivas en el Perú contemporáneo.

La limitada demanda de mano de obra local por la minería tecnificada ha generado nuevos retos para la convivencia entre las empresas y los ciudadanos ${ }^{8}$. Las demandas se han apartado del esquema de empleados y empleadores para centrarse

\footnotetext{
Recojo el término de Sabatier (1998).

8 Mientras que las empresas mineras utilizaban mano de obra local a gran escala, las demandas estuvieron lideradas por sindicatos de trabajadores que exigían mejorar las condiciones laborales y beneficios colectivos (Kruijt y Vellinga, 1983).
} 
en beneficios directos otorgados por la empresa minera a la población local, como compensación por la actividad y por el acceso a los recursos naturales (Damonte, 2013; Zavaleta, 2014; Li, 2017) $)^{9}$. Por ejemplo, en sus primeros ocho años de operaciones, Yanacocha había invertido más de US\$ 2000 millones en la construcción de sus instalaciones, lo cual incluía la apertura de nuevos caminos, la extensión de la red de tendido eléctrico y la construcción de sistemas de agua potable (Bury, 2007b, p. 261). A la par, la empresa modificaba los cursos de canales de riego y flujos de agua, y diariamente removía toneladas de tierra que alteraron de manera radical la geografía de la región. De esta forma, los impactos de las actividades de la empresa en los modos de vida y de producción de la población rural fueron de carácter heterogéneo.

Sobre la base del análisis de tres centros poblados con impactos altos, medios y bajos por las actividades de la empresa, Bury (2007b) encuentra hacia el año 2005 que los centros poblados que habían mantenido contacto con la empresa se habían beneficiado de la inversión en infraestructura, acceso a servicios de educación y salud, y programas para mejorar la productividad otorgados por la mina como parte de proyectos de responsabilidad social que mostraron impactos positivos en índices como nutrición, creación de capacidades y cobertura de servicios básicos. No obstante, el mismo análisis concluye que las actividades mineras tuvieron impactos negativos en el acceso a los recursos naturales, principalmente agua potable, recursos de vertiente y abastecimiento de agua para riego ${ }^{10}$.

El deterioro de las fuentes de agua enfrentó paulatinamente a la población rural con la empresa (Tanaka y Meléndez, 2009). La percepción de que el agua estaba siendo contaminada por Yanacocha se extendió rápidamente y, como consecuencia directa, se le responsabilizó por la propagación de enfermedades, muerte de animales y baja productividad agrícola (Bury, 2007b, p. 280). Por otra parte, la compra de grandes extensiones de terreno por la empresa aumentó el precio de la tierra, lo cual generó incentivos para convertir las propiedades en otras formas de capital (Bury, 2007b, p. 286). Debido a que el aumento constante en los terrenos restaba la capacidad de invertir los ingresos en la compra de nuevas tierras, los campesinos optaron por invertir principalmente en cabezas de ganado y cultivos mejorados (Bury, 2007, p. 286) o en la adquisición de vehículos de transporte o maquinaria que pudieran servir para ser rentados a Yanacocha.

\footnotetext{
9 Si bien existe un canal institucional para redistribuir las ganancias de la inversión —el canon destinado a los gobiernos subnacionales-, estos ingresos tardan en ser convertidos en proyectos de inversión pública (Barrantes et al., 2012, p. 63), por lo que para la población rural existen mayores incentivos para negociar directamente con las empresas en lugar de hacerlo con los gobiernos subnacionales.

10 Un diagnóstico similar puede verse en Meléndez (2009) y Tanaka y Meléndez (2009).
} 
Visto de manera longitudinal, resulta paradójico que la apertura de caminos, programas sociales de tecnificación agropecuaria y capitalización de los campesinos haya permitido elevar la productividad del campo a la par que los recursos hídricos eran deteriorados por la actividad extractiva y la tierra se perjudicaba debido a mayor presión productiva en cortas extensiones de terreno. A pesar de que la minería fue directa o indirectamente responsable del incremento productivo, la población podría haber tendido a evaluar las consecuencias negativas de los impactos en los recursos naturales, e incluso considerarlos como más perjudiciales, ya que iban en contra del reciente incremento en las ganancias ${ }^{11}$.

Por otra parte, como apuntan Barrantes, Cuenca y Morel (2012, p. 152) en relación con el ingreso de Lumina Cooper a la región en ańos posteriores, la compra de maquinarias o vehículos de carga y transporte por los minifundistas que vendieron sus terrenos a la empresa representó un cambio en su actividad económica, de la producción directa a la renta. Lamentablemente, esta inversión se realiza sobre la base de activos que se deprecian rápidamente y con altos costos de mantenimiento. No obstante, la conformación de empresas de servicios es una de las estrategias más comunes de la población rural para vincularse con el núcleo productivo. Muestra de ello es que, de acuerdo con Barrantes y sus colaboradores, la capitalización y posterior aparición de empresas de servicios ha sido la dinámica básica de la influencia de la minería en la diversificación económica.

\section{Gráfico 1. Dinámica de la influencia de la minería en la diversificación} económica de Cajamarca

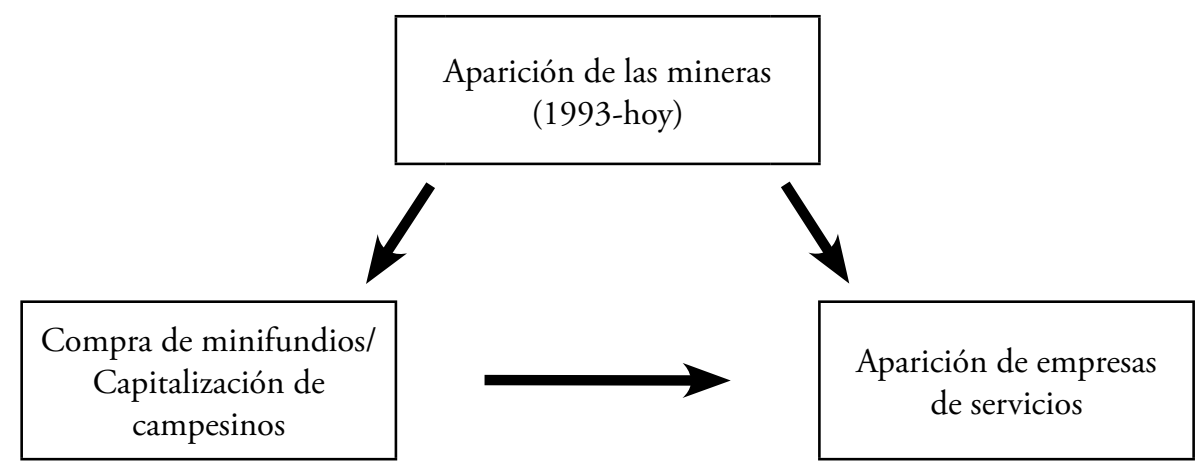

Fuente: Barrantes, Cuenca y Morel (2012).

11 Utilizando datos provenientes del Censo Agrario 2012, Orihuela, Huaroto y Paredes (2014) encuentran que la presencia de actividades mineras está asociada con un alto porcentaje de agricultores que señala que el agua de riego está contaminada o es escasa. 
La constitución de empresas para brindar servicios a un solo demandante limita de manera drástica el mercado y, naturalmente, frente a la abundancia de oferta, reduce el precio de los servicios. Estas condiciones no solo configuran un escenario de alto riesgo para la inversión de capital en este tipo de empresas, sino que fomentan los conflictos. De acuerdo con un consultor de Yanacocha, durante el primer estudio que realizó, en el año 2004, se obtuvo que la minera había firmado acuerdos para contratar maquinaria y vehículos de transporte con cerca de cuatrocientas empresas comunales, pero solo había contratado, al menos una vez, a poco más de treinta ${ }^{12}$. Esto generaría obstáculos importantes para su posterior expansión. En el mediano plazo, la percepción de que los costos asociados al deterioro de los recursos naturales eran mayores que los beneficios recibidos a través de programas sociales y contratación de empresas subsidiarias elevó los costos de transacción entre la población y la empresa. El conflicto de Combayo ilustra de buena manera este fenómeno.

\section{Combayo}

En agosto de 2006, comuneros del centro poblado Combayo, en el distrito de La Encañada, iniciaron una protesta frente a las instalaciones de la empresa y bloquearon la carretera de acceso principal de la ciudad de Cajamarca. El motivo central de la movilización era el incumplimiento de contratos establecidos entre Yanacocha y los comuneros, principalmente el retraso en la construcción de la carretera Puente Otuzco-Combayo (en la cual se preveía la contratación por la empresa de trabajadores del centro poblado), y como demanda adyacente, atender los impactos ambientales producidos por la ampliación del yacimiento Carachugo en la cuenca del Mashcon (Caballero, 2012, p. 13). Frente a la negativa de Yanacocha a negociar si previamente no se suspendía la movilización, la protesta se radicalizó y los manifestantes ingresaron a las instalaciones de la mina, lo cual terminó con la muerte de uno de los comuneros a manos de agentes de seguridad de la compañía (Caballero, 2012, p. 14).

Si bien la discordia se originó con el incumplimiento de acuerdos, rápidamente el conflicto devino en la oposición a la ampliación de las actividades extractivas sin que se garantizase la calidad y cantidad de agua de la cuenca, para lo cual se solicitó la realización de un estudio de balance hídrico ${ }^{13}$. Transcurridos algunos años desde la instalación de Yanacocha en la zona, los ciudadanos fueron capaces de evaluar retrospectivamente los efectos de las actividades extractivas, lo que orientó las opciones de la población a disminuir los riesgos de afectación de los medios de

\footnotetext{
12 Informante 1, entrevista personal con el autor. Lima, 9/7/2012.

13 Para solucionar el conflicto fue necesaria la intervención de la Presidencia del Consejo de Ministros. En la mesa de diálogo instalada se acordó suspender el proyecto Carachugo II hasta esperar los resultados del estudio de balance hídrico, el cual finalmente nunca se realizó (Caballero, 2012, pp. 19-20).
} 
subsistencia. Un documento suscrito durante el preludio del proyecto Conga y en vísperas del conflicto de Combayo ilustra esta situación.

En abril de 2005, dirigentes y autoridades locales de Cajamarca y Celendín remitieron un oficio a la Dirección General de Asuntos Ambientales del Ministerio de Energía y Minas (Minem) en el que solicitaban que se declarase inviable el proyecto Conga y la ampliación de Carachugo (Combayo). En los párrafos tercero y cuarto señalan lo siguiente:

TERCERO: Son más de una docena de ańos que tenemos la presencia de Minera Yanacocha en la jurisdicción de La Encañada y hoy estamos más pobres que cuando empezó a sacar oro esta transnacional, como lo demuestran las estadísticas, para muestra lo que la Municipalidad de La Encañada [recibió] de canon el año 2003. Es 16 céntimos de nuevo sol por poblador diario, y si aun así nos dieran los 7 millones de dólares que tanto pregonaba [la empresa], solamente llega a dos soles diarios por poblador, pero a cambio recibimos aguas ácidas, como lo demuestra las aguas del área de Combayo y condenamos a la agricultura y la ganadería a desaparecer.

CUARTO: No podemos permitir que nuestras aguas, lagunas y ríos desaparezcan y se contaminen con el engaño de que nos darán trabajo o empresa, como lo viene haciendo a la actualidad a cambio de dejar desaparecer la laguna Challuagón, laguna el Perol, laguna Azul, laguna Cortada, laguna Pencayoc y otras, ya que solamente dan trabajo a 6500 personas como lo dicen en el EIA ${ }^{14}$ de la ampliación Carachugo II, que no se espera que el proyecto cree nuevos puestos de trabajo.

La formulación del oficio es un ejemplo concreto de la racionalidad de la población rural de Cajamarca y la experiencia acumulada de relacionamiento con Yanacocha. En primer lugar, muestra que los recursos derivados de canon no son percibidos como un beneficio significativo por su carácter indirecto. Es ilustrativo que para evaluar su importancia no se analiza su utilización en la generación de valor público por los gobiernos locales, sino que se realiza un cálculo de su asignación per cápita (diario). Luego de ello, se establece que los beneficios recibidos por este concepto resultan insuficientes y, en lugar de ganancias monetarias, la mina ha generado pérdidas relacionadas con los recursos naturales (aguas ácidas) lo cual representa efectos negativos para las actividades agropecuarias. En segundo lugar, se menciona explícitamente que no se justifica aceptar la oferta de trabajo de la empresa a cambio de afectar una serie de lagunas. Nuevamente, se realiza una comparación entre los costos y los beneficios a partir de la cual se concluye que el nuevo

14 Estudio de impacto ambiental. 
proyecto no generará nuevos puestos de trabajo, por lo cual sería un mal acuerdo aceptar el desarrollo de las nuevas actividades extractivas bajo esas condiciones.

En un trabajo reciente, Fabiana Li (2017) argumenta que la negociación con la empresa con las comunidades campesinas del área de influencia de Yanacocha «a menudo queda fuera de la lógica de mercado del cálculo racional» $(2017$, p. 45). Sin embargo, los casos presentados por la propia autora difícilmente sostienen esa aseveración. En uno de los capítulos centrales de su libro, Li toma como ejemplo de la negociación con Yanacocha la afectación del canal Túpac Amaru, en la comunidad de Tual, el cual fue construido en la década de 1980 para facilitar el pastoreo de ganado vacuno destinado a la producción lechera.

Luego de que la empresa estableciera un programa de compensación monetaria por su afectación a la calidad del agua del canal, el padrón de usuarios se incrementó de 205 personas en 2002 (cuando inició el programa) a 650 en 2011 ( $\mathrm{Li}, 2017$ ), lo cual derivó en la fragmentación extrema de las horas de regadío asignada a cada usuario (que dividían en una hoja de Excel) ${ }^{15}$. Así, la comunidad estableció un sistema informal de distribución de los recursos derivados de la compensación al dividirlo en partes iguales entre todos aquellos que ingresaban al padrón. Ello muestra que los comuneros de Tual, de la misma manera que los dirigentes firmantes del oficio presentando anteriormente, comprendieron la compensación de manera igualitarista y racional: la distribución individual e igualitaria del monto total asignado a la comunidad basada en un cálculo matemático ${ }^{16}$.

Como ambos casos muestran, los campesinos de Cajamarca establecieron negociaciones pragmáticas que buscaban cuantificar la afectación de los medios de subsistencia y reclamar su compensación. Por ello, la paulatina percepción de que los costos ambientales eran más altos que los beneficios materiales directamente recibidos incrementó la aversión al riesgo frente a las nuevas incursiones de la empresa. Como veremos a continuación, en ese escenario (post Combayo), el adelanto de beneficios y el establecimiento de acuerdos previos adquirieron una mayor relevancia, impulsando a la compañía a incrementar su gasto social ${ }^{17}$.

\footnotetext{
15 Es importante destacar que, a mediados del siglo XX, la parcelación de las haciendas de Cajamarca y el crecimiento de la industria lechera dinamizaron el campo, involucrando a la mayoría de la población rural de la provincia en el mercado laboral (Deere, 1990). En ese sentido, desde hace décadas predominan los estándares de transacción monetaria.

16 Desde un caso distinto, Asensio (2016) ha llamado este tipo de (re)distribución «keynesianismo andino".

17 Solo entre 2006 y 2008 la empresa destinó más de S/ 267 millones a través del Fondo Solidaridad con Cajamarca a proyectos de desarrollo en la región en las áreas de salud, nutrición, educación e infraestructura.
} 


\section{Conga}

Con la vida útil del yacimiento Yanacocha en expiración, Minas Conga, un proyecto polimetálico valorizado en US\$ 4800 millones en inversión, constituía el proyecto expansivo más importante de la empresa ${ }^{18}$. El ingreso a la nueva zona (fuera del «área tradicional» de actividades de la empresa) supuso una estrategia de relacionamiento previo que transcendiera la compra de tierras, como fuera en 1992. El Proceso de Participación Ciudadana (PPC) del EIA para el subsector minero, obligatorio desde el año 2008, constituyó un espacio de negociación entre la población y Yanacocha, el cual ilustra en buena manera los intereses y preocupaciones centrales de la población rural y la connotación política que adquirió el proceso ${ }^{19}$. Como resultado de la estrategia, 32 caseríos del área de influencia directa del proyecto aprobaron el EIA en una audiencia pública.

A partir de enero de 2009, el equipo de Asuntos Externos de Yanacocha, en coordinación con la agencia de publicidad Toronja, inició el proceso de elaboración de una estrategia de relacionamiento que permitiese presentar el proyecto ante los ciudadanos dentro del área de influencia directa en el marco del Plan de Participación Ciudadana. Además de brindar información en asambleas comunales y talleres participativos, la estrategia diseñada por los agentes de la empresa consistía en generar vínculos con las comunidades del área a través de acuerdos que comprometiesen la participación ciudadana. Estos talleres informativos se desarrollaron en 32 caseríos del área de influencia directa, con los cuales el equipo de negociación estableció una serie de "preacuerdos» que permitirían atender las demandas inmediatas de la población en materia de infraestructura, mejoramiento productivo y almacenamiento de agua.

Realizar este trabajo era de central importancia para Yanacocha, dado que era consciente de las connotaciones políticas que podría generar el plan de acción del proyecto. De acuerdo con el EIA, las lagunas Mala, Azul, Chica y Perol serían destruidas para explotar el mineral o servir como depósitos de residuos y sus aguas trasvasadas a reservorios artificiales que, de acuerdo con el estudio, permitieran almacenar una mayor cantidad de agua y cosechar lluvias. En tal sentido, era prioritario para la empresa resaltar los beneficios de los reservorios frente a la desaparición de las lagunas.

18 El descubrimiento de los yacimientos mineros de Chailhuagón y Perol, que conforman el proyecto Conga, se realizó en 1991 por la Compañía de Exploraciones, Desarrollo e Inversiones Mineras (CEDIMIN SAC). Entre 1994 y 2004 la compañía exploró los yacimientos hasta que fue adquirida por Minas Buenaventura, socia minoritaria de Yanacocha (Knight Pièsold, 2010).

19 De acuerdo con la Resolución Ministerial No 304-2008-MEM-DM, durante la elaboración del EIA el titular minero deberá coordinar con la autoridad competente en la región la realización de al menos un taller participativo y cualquier otro de mecanismo de participación ciudadana con la finalidad de informar respecto de los avances y resultados en la elaboración del EIA y registrar los intereses de la población involucrada, los aportes, comentarios y observaciones. 


\section{Cuadro 1. Preacuerdos de Yanacocha con caseríos del área de influencia}

\begin{tabular}{|c|c|c|}
\hline N. ${ }^{\circ}$ & Caserío & Preacuerdo \\
\hline 1 & San Nicolás & $\begin{array}{l}\text { Evaluación de alternativas de represamiento de agua: laguna Chailhuagón, } \\
\text { Bofedal, Pampa Chica-Huayramachay, laguna Cahire }\end{array}$ \\
\hline 2 & & Evaluación para la instalación de microrreservorios: Poyo Secreto o Pipirija \\
\hline 3 & & Vivero forestal, producción de 20000 plantones \\
\hline 4 & & Riego tecnificado por aspersión: Piloto Reservorio El Lirio \\
\hline 5 & & Asistencia técnica agropecuaria \\
\hline 6 & & $\begin{array}{l}\text { Evaluación para instalación de planta de transformación de leche de } \\
\text { capacidad de mil litros/día }\end{array}$ \\
\hline 7 & Agua Blanca & Riego tecnificado \\
\hline 8 & & Mejoramiento de pastos (instalación de un piloto) \\
\hline 9 & & Planta quesera de capacidad de 500 1/día \\
\hline 10 & & Botiquín veterinario \\
\hline 11 & & Botiquín de salud \\
\hline 12 & Quengorío Alto & Preocupación previa por el tema ambiental antes de la priorización \\
\hline 13 & Lagunas de & Diseño y evaluación de proyecto de agua potable \\
\hline 14 & Combayo & Formulación y evaluación de proyecto de riego por aspersión \\
\hline 15 & & Estudios carretera Lagunas-Quinuapampa \\
\hline 16 & Piedra Redonda/ & Agua \\
\hline 17 & El Amaro & Alfabetización \\
\hline 18 & & Mejoras de vías de comunicación \\
\hline 19 & & Desarrollo ganadero \\
\hline 20 & & Riego tecnificado \\
\hline 21 & Huasiyuc & Agua \\
\hline 22 & Jadibamba & Alfabetización \\
\hline 23 & & Mejoras de vías de comunicación \\
\hline 24 & & Desarrollo ganadero \\
\hline 25 & & Riego tecnificado \\
\hline 26 & Quengorío Bajo & Agua potable: mano de obra y algunos materiales \\
\hline 27 & & Cerco perimétrico del colegio \\
\hline 28 & & Mercado: servicios higiénicos y pozo séptico \\
\hline 29 & & Construcción del puente Quengorío \\
\hline 30 & & Nivelamiento de la Plaza de Armas \\
\hline 31 & & Nivelamiento del estadio \\
\hline 32 & Chugurmayo & Apoyo en la implementación del centro de salud \\
\hline 33 & & Apoyo en la implementación del centro educativo \\
\hline 34 & & Proyecto integral de desarrollo ganadero \\
\hline
\end{tabular}

Fuente: Knight Piésold Consulting (2010). 
Si bien el PPC se encuentra diseñado para brindar información sobre las características del proyecto y generar consenso con respecto a las estrategias de mitigación de impactos ambientales, en el caso específico de Conga el proceso transcendió estas funciones y constituyó un espacio de negociación: la empresa aseguró a la población que la calidad y cantidad del agua no serían alteradas y ofreció solucionar demandas específicas no relacionadas con la actividad extractiva. Como resultado, los «preacuerdos» derivados del proceso tuvieron poco que ver con el EIA, pero sí con el aumento de la producción agrícola (ver cuadro 1). En tal sentido, la población apoyó la ejecución del proyecto en la medida en que se adelantaron beneficios capaces de dinamizar la economía rural ${ }^{20}$.

Dentro del área de influencia, la estrategia desarrollada por Yanacocha generó resultados positivos, ya que parecía asegurar un clima de paz social para el inicio del proyecto. Un consultor externo de la empresa, quien participó de forma directa en el ingreso de Yanacocha a la nueva zona, narra el proceso de la siguiente manera:

Cuando se trabajó el EIA de Conga en los 32 caseríos, lo interesante fue que fue una propuesta que los caseríos aprobaron porque se trabajó muy bien. Además, se decía: «Compañeros, vamos a vaciar una laguna, porque ahí está el mineral, porque ahí va a estar la operación minera, pero el agua la vamos a trasvasar a las quebradas vecinas y vamos a hacer de estas lagunas, lagunas más grandes. Con un dique natural de piedras y material de la zona, vamos a hacer reservorios que repliquen las lagunas. ¿̇probado?». Todas las asambleas, está firmado: «aprobado» [...]. Entonces, cuando llegamos a los talleres ya formales del EIA y a la audiencia del EIA, los dirigentes ya sabían la propuesta, la gente ya sabía la propuesta, y ya se había comenzado a ejecutar programas de salud; por ejemplo, nutrición infantil, que la gente valora ${ }^{21}$.

En marzo de 2010 se llevó a cabo la Audiencia Pública del proyecto Conga en el caserío San Nicolás de Chailhuagón, ubicado en el distrito de La Encañada, la cual contó con la participación aproximada de 3000 personas y un número significativo de autoridades políticas, entre las que se encontraban el entonces gobernador regional, Jesús Coronel; los alcaldes provinciales de Celendín y Cajamarca, y los alcaldes distritales de Sorochuco, Huasmín y La Encañada. La asamblea se llevó a cabo sin incidentes y el EIA fue aprobado de manera simbólica por los asistentes. Según el testimonio del informante, el evento cosechaba el trabajo realizado por el equipo de Yanacocha:

\footnotetext{
20 A ello se sumó la implementación de programas sociales privados en salud y nutrición infantil desde 2010. Informante 1, entrevista personal con el autor, Lima, 9/7/2012.

21 Informante 1, entrevista personal con el autor, Lima, 9/7/2012.
} 
Lo que nosotros hicimos es trabajar duro con cada caserío, no solamente en negociar proyectos de desarrollo, sino también repetir tantas veces el rollo de forma que, cuando lleguen a la audiencia, sea un evento oficial que cierre un proceso, no que lo abre, porque si no es torpe. Entonces, termina siendo eso, un evento más protocolar. ¿Qué es lo que estás demostrando ahí? Que tienes apoyo social. Si en esos 32 caseríos suman cerca de 1500 familias y tienes 3000 personas asistentes al evento y mil más afuera escuchando, estás recontra cubierto. Está claro que con 3000 personas no has ido a hacer una exposición técnica con debate, sino a cosechar un trabajo técnico ${ }^{22}$.

Esa opinión coincide con otro informante, miembro del equipo de relaciones comunitarias del proyecto Conga, quien afirma: "Tú no vas a una asamblea de 4000, 5000 personas para consultar, tú vas para ganar» ${ }^{23}$, lo cual confirma la dimensión política con la cual Yanacocha entendió el proceso. Los operadores políticos que posteriormente liderarían las protestas contra el proyecto criticaron las asambleas en su momento, argumentado que estas no expresaban la verdadera voluntad de los ciudadanos porque habían sido cooptados a través de promesas de contratos y beneficios. Milton Sánchez, presidente de la Plataforma Interinstitucional de Celendín, expresa lo siguiente:

La audiencia pública la presentaron en una comunidad, Nicolás de Chailhuagón, que pertenece a La Encañada. Si tú ves el mapa del proyecto, más del ochenta, noventa por ciento está dentro del territorio de Celendín, pero lo hicieron estratégicamente en Chailhuagón porque pertenece a La Encañada y en La Encañada ya son dieciocho ańos de actividad minera y les habían ofrecido a los pobladores que ahí iban a poner sus empresas $[\ldots]^{24}$.

Más allá de sus discrepancias sobre la naturaleza del proceso, los testimonios de las personas relacionadas con la empresa, como los de aquellas que se opondrían al proyecto Conga, coinciden en que tuvo una connotación visiblemente política. Esto es resaltante, porque para la empresa cumplir este tipo de funciones genera costos que, en una lógica puramente económica, preferiría evitar. No obstante, las empresas en general -y Yanacocha en específico- se encuentran impulsadas a desarrollar una dimensión política bajo el peso de su propia estructura (gran capacidad económica y leve demanda de mano de obra local), a lo que se suman las falencias institucionales del Estado peruano ${ }^{25}$.

22 Informante 1, entrevista personal con el autor, Lima, 9/7/2012.

23 Informante 2, entrevista personal con el autor, Cajamarca, 20/6/2012.

24 Milton Sánchez, entrevista personal con el autor, Cajamarca, 20/6/2012.

25 La provincia de Celendín, en la cual se concentra el proyecto, se ubicaba en el cuartil inferior de las 196 provincias del país tanto en el Índice de Desarrollo Humano (IDH) como en el Índice de Densidad del Estado (PNUD, 2009), mientras que para 2011 la pobreza alcanzaba 66,3\%. 
Bajo esta perspectiva, no es sorprendente que un proceso formal cuyo objetivo es armonizar las relaciones entre las grandes empresas y la población haya sido reestructurado por la práctica. Los objetivos que inspiran el proceso, como el acceso a la información y diálogo, son reconfigurados por la asimetría natural entre ambas partes. Para los ciudadanos fue una oportunidad específica para obtener beneficios que el Estado no ha sido capaz de brindarle, y tecnología - como riego tecnificado y microrreservorios de agua - que permita aumentar la productividad agrícola. En tal sentido, los beneficios fueron evaluados como superiores a los costos representados por la incertidumbre producida por los cambios en la geografía. Por su parte, para Yanacocha fue el escenario preciso para ganar por puesta de mano a las organizaciones sociales que se oponían a la minería en la región (en ese momento aletargadas), estableciendo acuerdos que garantizasen apoyo a su proyecto de expansión más importante.

Los preacuerdos firmados con las poco más de treinta comunidades dentro del área de influencia permitieron a la empresa mostrar - luego de mucho tiempocredenciales de licencia social. Sin embargo, no contaba con que un proceso de connotaciones políticas opuestas se gestaba en paralelo. La Plataforma Interinstitucional de Celendín, una organización que cuestionó públicamente la viabilidad del proyecto, inició un trabajo de difusión en la zona rural cercana a Conga, consiguiendo atención en los caseríos que no habían sido incluidos en el proceso de participación. Como se ilustra en la siguiente sección, estas poblaciones veían amenazados sus medios de subsistencia sin haber establecido vínculos con la empresa o arribado a acuerdos. El EIA establecía que, para realizar el proyecto, cuatro lagunas sería eliminadas, lo cual era un asunto fácilmente politizable. Paradójicamente, la creación de reservorios artificiales, que había sido planificada como el principal mecanismo de mitigación ambiental, se transformó en el eje de la lucha política de los operadores opuestos al proyecto.

\section{CoAliciones Promotoras: de Quilish a Conga}

El conflicto en torno al cerro Quilish, ubicado a escasos kilómetros de la ciudad de Cajamarca, impulsó la creación de la primera "coalición promotora» en contra de la expansión de Yanacocha en el año 2004. A los comités de regantes y las rondas campesinas — actores rurales que habían sido hasta el momento la oposición social más visible al proyecto de expansión - se sumaron actores principalmente urbanos que cumplieron un papel importante en las manifestaciones: el Frente de Defensa de Cajamarca, creado durante la década de 1990, y un conjunto de ONG ambientalistas recientemente fundadas en la región, la más notable el Grupo de Formación e Intervención para el Desarrollo Sostenible (Grufides). Asimismo, la Municipalidad 
de Cajamarca cumplió un papel central en el conflicto al buscar convertir el área en zona protegida (Meléndez, 2009).

Desde Quilish en adelante, los conflictos contra la expansión de Yanacocha han movilizado ciudadanos sobre la base de un sustento esgrimido desde lo técnico (Zavaleta, 2013; Li, 2017; Yrrivaren, 2017). Con variantes, el argumento base argüido por las élites locales puede resumirse de la siguiente forma: el ecosistema de Cajamarca consiste en un sistema complejo de cerros, bofedales y lagunas, cuyas aguas se encuentran interconectadas de manera subterránea entre sí, por lo que cualquier alteración a una parte afecta a todo el conjunto. Esto ha llevado a que las controversias, en términos discursivos, se centren en la necesidad (o deficiencias) de documentos de gestión ambiental: el «estudio hidrobiológico» en el caso de Quilush; el «balance hídrico» en Combayo y EIA en Conga ${ }^{26}$.

En un primer momento, hacia principios de 2010, la oposición al EIA de Minas Conga y a la desaparición de las lagunas fue puesta en agenda por un grupo reducido de organizaciones sociales que buscaron establecer una corriente de opinión con respecto al proyecto, lo que fue el germen de una nueva "coalición promotora" (Sabatier, 1998; Cortez y Maillet, 2018).

Utilizo esta definición en la medida que nos permite distinguir las diversas articulaciones opositoras a la expansión de Yanacocha desde 2004 de los movimientos sociales y entenderlas como «agrupaciones de actores que se constituyen como sujetos políticos en torno a un cuerpo de creencias, sin que generen, necesariamente, compromisos orgánicos asociados a sus demandas» (Cortez y Maillet, 2018, p. 6). Si bien el discurso ambientalista se ha mantenido vigente durante más de una década, las coaliciones cambiaron de composición organizacional en cada ciclo de movilización. Mientras que en 2004 el Frente de Defensa Ambiental de Cajamarca (FDAC) lideró las protestas de Quilish, durante el conflicto en torno a Conga el CUL fue la organización articuladora de la oposición y el FDAC cumplió un papel marginal.

En marzo de 2010, luego de celebrada la asamblea pública en San Nicolás de Chailhuagón, se formó en Celendín la Coordinadora Interprovincial de Frentes de Defensa, que agrupaba inicialmente a cinco frentes de las provincias de San Marcos, Cajamarca, Celendín, Hualgayoc y San Pablo. De acuerdo con Wilfredo Saavedra — presidente del FDAC—, durante la cuarta asamblea de este organismo

\footnotetext{
26 La politización de los aspectos técnicos vinculados con la gestión ambiental da buena cuenta del grado de "glocalización» que adquirió el conflicto minero en Cajamarca. Actores transnacionales dotaron de recursos materiales y discursivos a los actores locales, pero también - cumpliendo lo observado por Paredes (2016) en los casos de Tambogrande y Espinar- dificultaron la formación de una plataforma regional de carácter duradero, al incentivar (sin quererlo) las alianzas entre comunidades específicas y actores internacionales antes que entre las mismas comunidades afectadas.
} 
de coordinación se acordó pedir la inviabilidad de Conga por daños irreversibles al medio ambiente. No obstante, sería una nueva organización, la Plataforma Interinstitucional de Celendín (PIC), estrechamente relacionada con Grufides, la que cumpliría un papel fundamental en difundir los impactos, a su juicio perjudiciales e irreversibles, que ocasionaría el inicio de las operaciones mineras. Milton Sánchez, presidente de la PIC, explica este proceso de la siguiente forma:

El trabajo que empezamos a hacer desde la plataforma fue informar a la gente, no oponernos sino informar a la gente. Y la gente no sabía lo que significaba. $Y$ ahí verdaderamente actuábamos bajo un principio: lo que uno no conoce no lo va a defender. Nadie sabía de la existencia de las lagunas arriba. Empezamos a rebotar por internet, por los medios de comunicación, y en las charlas que nos íbamos comunidad por comunidad, nos hemos ido a mostrarles a través de diapositivas qué era el proyecto Conga y qué iban a destruir y de dónde venía el agua. Entonces, la gente comenzó a preocuparse, se preocupó tanto que se instalaba ya en el escenario de Celendín como un tema de coyuntura.

Como se ha indicado a lo largo del artículo, la población rural es muy sensible a la posibilidad de perder acceso a las fuentes de agua o a su disminución en cantidad y calidad, especialmente si estos costos potenciales son asumidos de manera «gratuita». Debido al trabajo realizado durante el proceso de participación por la empresa, la PIC tuvo dificultades para ingresar a los 32 caseríos incluidos dentro del área de influencia directa estipulada por Yanacocha. Sin embargo, en aquellos caseríos cercanos que no habían sido incluidos en el proceso participativo (y que no llegaron a acuerdos con la empresa), el trabajo político de la PIC generó resultados, principalmente porque se vieron amenazados por la posibilidad de que las aguas fueran contaminadas o disminuidas sin recibir ningún tipo de beneficio en compensación por el riesgo latente ${ }^{27}$. Cuando el conflicto estalló, en octubre de 2011, la población de estos caseríos se movilizó rápidamente y constituyó el núcleo central de la primera marcha hacia las lagunas que pretendían ser reemplazadas por reservorios.

La multiplicidad de actores que se involucraron en el preludio de conflicto sostenía lógicas y estrategias diferenciadas. Por una parte, se encontraban actores más politizados, los cuales proponían una defensa férrea del medio ambiente en contra de cualquier actividad minera en las cabeceras de cuenca. Por otra, las autoridades de los centros poblados estaban preocupadas concretamente por el futuro de las lagunas y acuíferos de la zona, mientras que otras organizaciones rurales

27 Informante 5, entrevista personal con el autor, Cajamarca, 22/6/2012; informante 8, entrevista personal con el autor, Cajamarca, 22/6/2012; informante 9, entrevista personal con el autor, Cajamarca, 24/6/2012. 
consideraban pertinente realizar nuevas exigencias a la empresa para operar, relativas a empleo y beneficios sociales. Por ejemplo, en abril de 2011 el comité de empresas comunales de Sorochuco realizó una asamblea en la cual se acordó exigir a Yanacocha seguro médico, capacitación para los comuneros, asfaltado de la carretera hacia Michiquillay y becas educativas (De Echave y Diez, 2013).

Meses después, a principios de octubre, el alcalde del distrito de La Encañada convocó a un paro indefinido para exigir a la compañía el cumplimiento de una serie de acuerdos que involucraban acciones de remediación ambiental y construcción de obras de infraestructura para la jurisdicción. De acuerdo con la autoridad, la empresa no había cumplido con los acuerdos y había destinado los fondos de su programa de responsabilidad social a otros distritos del departamento. El estallido de la violencia en La Encañada daría inicio a las protestas más contundentes y duraderas de la historia de la región. Un paro departamental indefinido iniciado el 24 de noviembre, convocado por el gobernador regional y el conjunto de organizaciones que demandaban asuntos a la empresa, tendría como resultado la suspensión del proyecto minero Conga, la declaración del estado de emergencia en cuatro provincias y la renuncia del presidente del Consejo de Ministros ${ }^{28}$.

Si bien el proyecto fue suspendido tras el aluvión de protestas, luego de la declaratoria de estado de emergencia, en diciembre de 2011, los principales líderes de la movilización decidieron conformar un organismo de coordinación entre los diferentes frentes, el CUL, que buscaba la cancelación definitiva del proyecto bajo el eslogan "Conga no va». La nueva coalición promotora, aglutinada en el CUL, se encontraba liderada por Milton Sánchez, presidente de la Plataforma Interinstitucional de Celendín (PIC); Edy Benavides, presidente del Frente de Defensa de los Intereses y Medio Ambiente de Hualgayoc/Bambamarca, e Ydelso Hernández, presidente del Frente de Defensa de los Intereses de la Región Cajamarca. Adicionalmente, agrupaba miembros de Grufides, la Central Única Nacional de Rondas Campesinas (Cunarc), la CGTP Cajamarca, SUTE-Cajamarca, la Federación Universitaria de Cajamarca y la Iglesia católica.

Las primeras dos organizaciones se encontraban limitadas a espacios geográficos específicos. La PIC era una organización conformada por una quincena de operadores aglutinados bajo el liderazgo de Milton Sánchez, quienes, durante la etapa

\footnotetext{
28 Desde el inicio de la protesta, el gobierno nacional buscó contener a los manifestantes y garantizar la ejecución del proyecto. A inicios de noviembre, una delegación conformada por los ministros de Ambiente, Agricultura y Energía y Minas arribó a la región para apaciguar el conflicto, sin éxito. El 4 de diciembre, el entonces presidente del Consejo de Ministros, Salomón Lerner, sostuvo una reunión con el gobernador regional y los líderes de la protesta sin lograr que depusieran el paro (Meléndez, 2015). El fracaso de las conversaciones derivó en la declaración de estado de emergencia y la renuncia de Lerner al cargo por mostrarse en desacuerdo con esta medida (Zavaleta, 2014).
} 
previa al conflicto fueron una suerte de cabecera de puente de Grufides para desarrollar actividades de incidencia política en los centros poblados y caseríos de Celendín ${ }^{29}$. Por su parte, el Frente de Defensa de Hualgayoc, heredero de los comités de defensa del medio ambiente formados a partir del año 2000, experimentó un proceso de descomposición en los años previos al conflicto y fue reactivado en 2010, cuando Edy Benavides —un profesor secundario- asumió la presidencia y decidió inscribirlo como una organización electoral. Su fortaleza principal en la coyuntura del conflicto fue convocar a las rondas campesinas de la provincia, las cuales cuentan con una importante fortaleza organizativa en las comunidades (Starn, 1999).

A diferencia de las organizaciones anteriores, el Frente de Defensa de los Intereses de la Región Cajamarca mostraba mayores niveles de organización. Como frente, su estructura era bastante precaria, pero se encontraba asentado sobre las organizaciones de la sociedad civil controladas por Patria Roja (PR). Formado tras una escisión del Partido Comunista a finales de la década de 1970 (Hinojosa, 1999), PR ha sido el único partido integrante de la alianza Izquierda Unida que logró sobrevivir al cisma del frente en 1989 y a la década de 1990, período en que las organizaciones partidarias se descompusieron (Tanaka, 1998). Su sobrevivencia, tanto en Cajamarca como en el resto del país, ha estado ligada al control político de gremios a través de los cuales opera. En efecto, si analizamos la composición de los integrantes del CUL, muchos de estos eran tanto líderes gremiales como cuadros de PR. Ejemplos son Humberto Boñón, ex secretario regional del Sutep; Vladimir Ruiz, ex presidente de la Federación de Estudiantes de la Universidad Nacional Cajamarca (FUC), y el propio Ydelso Hernández, presidente de la Cunarc.

El control del sindicato de maestros y la central de rondas campesinas fueron los pilares sobre los cuales PR pudo estructurar la movilización. Asimismo, la figura de Gregorio Santos — quien como gobernador regional y entonces militante de PR se plegó a la protesta - fue crucial para mantener con cierta vitalidad la protesta hasta mediados de 2012. Al intervenir directamente en el conflicto, Santos hábilmente se posicionó como antagonista del propio presidente de la República, Ollanta Humala, quien en noviembre de 2011 había afirmado que el proyecto era «importante para el Perú», agregando una dimensión adicional al conflicto: la del gobierno regional contra el gobierno central (Meléndez, 2015).

En este contexto, el fortalecimiento de presencia política de Santos ha sido el fenómeno electoral más importante de Cajamarca desde la descomposición del partido aprista en la región. En 2014, luego de su encarcelamiento preventivo por un presunto delito de corrupción (lo que fue publicitado por su movimiento como

\footnotetext{
29 Informante 6, entrevista personal con el autor, Cajamarca, 23/6/2012; informante 10, entrevista personal con el autor, Cajamarca, 24/6/2012.
} 
la venganza política por su oposición a Conga), fue reelegido gobernador con trece puntos porcentuales por encima de su primera elección. En los tres distritos que componen el área de influencia de Minas Conga, Santos obtuvo más del $50 \%$ de los votos, mientras que en las provincias de Celendín y Hualgayoc — las cuales fueron centrales en las movilizaciones antimineras- también superó el umbral del 50\%. Dos años después, como candidato presidencial, obtuvo resultados similares: mientras que recibió menos del 5\% de la votación nacional, en Cajamarca el 40\% de los electores votó por Santos para ocupar la presidencia.

En resumen, si bien las respuestas sociales a la minería en Cajamarca han permitido la articulación de un discurso político centrado en la protección del medio ambiente y, por otra parte, la proyección política de la figura de Gregorio Santos, esto no se tradujo en términos organizativos. El CUL languideció rápidamente, mientras la expulsión de Santos de PR en 2016 afectó la fortaleza organizativa del MAS, el movimiento regional que fundó en la región. Sin Santos como candidato, y sin poder liderar una propuesta de desarrollo alternativo para una de las regiones más pobres del Perú, el MAS perdió la gobernación en 2018 y obtuvo resultados ambivalentes en el resto de la región.

\section{Conclusiones}

Durante la primera década del siglo XXI, el alto precio de los metales promovió un nuevo ciclo de producción minera global. En el Perú, la industria generó importantes ingresos al Estado e impulsó el crecimiento de la economía a tasas incluso superiores al resto de países de América Latina. Sin embargo, a diferencia de la mayoría de los países de la región, los efectos redistributivos de la bonanza fueron limitados. Por una parte, estrictas políticas ortodoxas restringieron el gasto social - lo cual explica, en parte, el triunfo del candidato que proponía mayores cambios al modelo económico en la primera vuelta de las elecciones 2006 y $2011-$ y la capacidad del Estado en todos sus niveles para transformar los montos recaudados en valor público fue limitada.

En los niveles locales, el desarrollo de la minería y la distribución de los recursos derivados originaron conflictos de diversa intensidad. En algunos casos, se limitaron a protestas que solo recibieron una breve mención en los diarios locales, mientras que en otros resultaron en movilizaciones de gran magnitud que impactaron en la política nacional. La historia reciente de la región Cajamarca permite analizar este continuo y limitarlo al relacionamiento de la población con una empresa específica: Yanacocha. Entre 1992 y 2011, los conflictos en torno a la minera evolucionaron de controversias con la población rural por el uso de la tierra hasta paros de alcance regional destinados a evitar la expansión de la compañía. 
Cuando Yanacocha ingresó a la región, generó expectativas que se tradujeron en la búsqueda de beneficios derivados del nuevo núcleo productivo. Sin la posibilidad de involucrarse como mano de obra en las actividades mineras, la población desarrolló estrategias económicas y políticas para acceder a los beneficios indirectos de la inversión ${ }^{30}$. Las respuestas económicas han estado orientadas a la creación de empresas subsidiarias para proveer a las mineras de servicios como transporte, limpieza y construcción de obras pequeńas, las cuales garantizaban —al menos en teoría - mayores ganancias que las actividades agropecuarias. Por otra parte, el ingreso de la empresa generó incentivos a la población para negociar beneficios directos como programas sociales o construcción de infraestructura.

Sin embargo, la paulatina afectación de los medios de subsistencia originados por Yanacocha brindó incentivos para la resistencia a las actividades mineras. Transcurridos algunos años desde la instalación de la empresa en la zona, los ciudadanos fueron capaces de evaluar retrospectivamente los efectos de las actividades extractivas, lo cual orientó las opciones de la población hacia minimizar los riesgos de afectación del medio ambiente. La población rural es propensa a preferir la evasión de riesgos en la medida que estos puedan afectar sus medios de subsistencia; ello en consideración de que el deterioro de la calidad del agua, por ejemplo, reduce la productividad agrícola e impacta negativamente en la economía rural.

Cerca de cumplir veinte años desde el inicio de sus actividades en Cajamarca, Yanacocha enfrentaba el reto de llevar a cabo su proyecto expansivo más importante: Minas Conga. Debido a su desprestigio, la empresa incrementó su gasto social y buscó arribar a acuerdos con la población dentro de su área de influencia directa. Sin embargo, un proceso de connotaciones políticas opuestas se gestaba en paralelo. Desde 2010, el Frente de Defensa Ambiental de Cajamarca y la Plataforma Interinstitucional de Celendín cuestionaron públicamente la viabilidad del proyecto. En su mayoría integrados por actores urbanos, sus operadores establecieron vínculos con los caseríos cercanos al proyecto - pero fuera del área de influencia-, los cuales se encontraban inseguros frente a la posibilidad de que las aguas fueran contaminadas o disminuidas por la actividad extractiva sin recibir ningún tipo de beneficio en compensación del riesgo latente.

En esta coyuntura, sectores sociales diversos confluyeron en la movilización de mayor magnitud de la historia de Cajamarca, la cual se mantuvo con cierta vitalidad hasta mediados de 2012, tiempo en el cual funcionó el CUL. No obstante, si bien la movilización fue exitosa en lograr sus objetivos en el corto plazo, los actores involucrados no fueron capaces de articular un movimiento social o una única opción electoral. Aunque la figura política de Gregorio Santos se fortaleció notablemente,

\footnotetext{
30 Las coplas han sido la forma más conocida de resistencia cultural.
} 
el MAS —el movimiento que fundó- muestra resultados ambivalentes sin Santos en contienda.

En cierto sentido, Cajamarca ha sido el escenario de apertura y clausura de un ciclo minero de alta productividad en el Perú: en 1992 se inauguraba en la región el primer gran proyecto luego de décadas de estancamiento, y casi veinte ańos después las protestas sociales evitaron la explotación de un proyecto extractivo valorizado en US\$ 4800 millones. La suspensión de Conga coincidió con la paulatina depreciación de los metales, los cuales iniciaron el retorno a sus precios normales a partir del ańo 2012. Este período ilustra de buena manera el desarrollo de la «nueva minería» en el Perú y sus impactos sociales. Si bien el trabajo se encuentra centrado en un estudio de caso, ha buscado realizar contribuciones teóricas sobre este fenómeno.

\section{REFERENCIAS}

Arce, M. (2015). La extracción de recursos naturales y la protesta social en el Perú. Lima: PUCP. Arellano Yaguas, J. (2014). Minería sin fronteras. Conflicto y desarrollo en las regiones mineras del Perú. Lima: IEP, PUCP.

Asensio, R. (2016). Los nuevos incas. La economía política del desarrollo rural andino en Quispicanchi (2000-2010). Lima: IEP.

Barrantes, R., Cuenca, R. y Morel, J. (2012). Las posibilidades del desarrollo inclusivo. Lima: IEP.

Bates, R. (1976). Rural responses to industrialization: a study of village Zambia. New Haven y Londres: Yale University Press.

Bebbington, A. (2013). Industrias extractivas, conflicto social y dinámicas institucionales en la región andina. Lima: IEP, Cepes, Propuesta Ciudadana.

Bebbington, A. y Hymphreys Bebbington, D. (2009). Actores y ambientalismos: conflictos socio-ambientales en el Perú. Íconos. Revista de Ciencias Sociales, 35, 117-128. https://doi.org/10.17141/iconos.35.2009.371

Bury, J. (2007a). Neoliberalismo, minería y cambios rurales en Cajamarca. En A. Bebbington, Minería, movimientos sociales y respuestas campesinas: una ecología politica de las transformaciones territoriales (pp. 49-80). Lima: IEP.

Bury, J. (2007b). Minería, migración y transformaciones en los medios de subsistencia en Cajamarca, Perú. En A. Bebbington (ed.), Minería, movimientos sociales y respuestas campesinas: una ecología politica de las transformaciones territoriales (pp. 231-274). Lima: IEP.

Caballero, V. (2012). La negociación de conflictos sociales 2006-2010. Tres modelos para armar. Lima: Cisepa.

Cortez, M. y Maillet, A. (2018). Trayectoria multinivel de una coalición promotora e incidencia en la agenda política nacional. El caso del conflicto de Pascua Lama y la ley de glaciares de Chile. Colombia Internacional, 94, 3-25. https://doi.org/10.7440/ colombiaint94.2018.01 
Damonte, G. (2013). Transformación de la representatividad política local en contextos extractivos a gran escala en los Andes peruanos. Revista Iberoamericana, 24(1), 65-104.

De Echave, J. y Diez, A. (2013). Más allá de Conga. Lima: RedGE.

De la Flor, P. (2014). Mining and Economic Development in Peru. Harvard Review of Latin America, 13(2), 24-27.

Deere, C.D. (1990). Household and class relations: peasants and landlords in northern Peru. Berkeley: University of California Press.

Defensoría del Pueblo (2017). El valor del diálogo. Lima: Defensoría del Pueblo.

Hinojosa, I. (1999). Sobre parientes pobres y nuevos ricos: las relaciones entre Sendero Luminoso y la izquierda radical peruana. En S. Stern (ed.), Los senderos insólitos del Perú: guerra y sociedad, 1980-1995 (pp. 73-92). Lima: IEP, UNSCH.

Irwin, A. y Gallagher, K. (2012). Chinese Investment in Peru: A Comparative Analysis. The Working Group on Development and Environment in the Americas.

Knight Piésold Consulting (2010). Minera Yanacocha, Proyecto Conga. Estudio de Impacto Ambiental: Plan de Participación Ciudadana. Lima.

Krujit, D. y Vellinga, M (1983). Estado, clase obrera y empresa transnacional. El caso de la minería peruana, 1900-1980. México: Siglo Veintiuno.

Li, F. (2017). Desenterrando el conflicto. Empresas mineras, activistas y expertos en el Perú. Lima: IEP.

Meléndez, C. (2009). Movilización sin movimientos. El caso de los conflictos entre comunidades y la empresa minera en Cajamarca. En R. Grompone y M. Tanaka (eds.), Entre el crecimiento económico y la insatisfacción social (pp. 321-380). Lima: IEP.

Meléndez, C. (2012). La soledad de la politica. Lima: Mitin.

Meléndez, L. (2015). Gobernar en tiempos de conflicto: conflictividad, accountability y autoridades subnacionales en el norte del Perú (2011-2014). Tesis de maestría, Flacso Ecuador.

Monaldi, Francisco (2014). The Mining Boom in Latin America. Harvard Review of Latin America, 13(2), 6 -8.

Orihuela, J.C., Huaroto, C. y Paredes, M. (2014). Escapando de la maldición de los recursos local: conflictos socioambientales y salidas institucionales. Lima: CIES.

Paredes, M. (2016). The glocalization of mining conflict: Cases from Peru. Extractive Industries and Society, 3(4), 1046-1057. https://doi.org/10.1016/j.exis.2016.08.007

Perla, C. (2017). Encuentro entre empresas mineras y poblaciones locales: usando las armas de James C. Scott. En P. Drinot (ed.), El Perú en teoría (pp. 107-136). Lima: IEP.

Perla, C. (2010). ¿Empresas mineras como promotoras del desarrollo? La dimensión política de las inversiones sociales mineras en el Perú. En C. Meléndez y A. Vergara (eds.), La iniciación de la politica (pp. 245-272). Lima: PUCP.

Sabatier, P. (1998). The Advocacy Coalition Framework: Revisions and Relevance for Europe. Journal of European Public Policy, 5(1), 98-130. https://doi.org/10.1080/ 13501768880000051 
Starn, O. (1999). Nightwatch: The Politics of Protest in the Andes. Durham, NC: Duke University Press. https://doi.org/10.1215/9780822382782

Tanaka, M. (1998). Los espejismos de la democracia: el colapso del sistema de partidos en el Perú. Lima: IEP.

Tanaka, M. y Meléndez, C. (2009). Yanacocha y los reiterados desencuentros: gran afectación, débiles capacidades de acción colectiva. En J. De Echave, A. Diez, L. Huber, B. Revesz, J. Ricard Lanata y M. Tanaka (eds.), Minería y conflicto social (pp. 73-97). Lima: IEP.

Yrrivaren, J. (2018). Ruido politico y silencio técnico. Un ensayo sobre la discusión socioambiental de Minas Conga. Lima: Punto Cardinal.

Zavaleta, M. (2013). La política de lo técnico: una aproximación al desarrollo del conflicto Conga. En J. Torres (ed.), Los limites de la expansión minera en el Perú (pp. 109-152). Lima: SER.

Zavaleta, M. (2014). La batalla por los recursos en Cajamarca. Lima: Cisepa-PUCP. 